\title{
Creatine Kinase MB to Total Creatine Kinase Ratio Measurement
}

National Cancer Institute

\section{Source}

National Cancer Institute. Creatine Kinase MB to Total Creatine Kinase Ratio

Measurement. NCI Thesaurus. Code C79441.

The determination of the ratio of creatine kinase MB compared to total creatine kinase present in a sample. The measurement may be expressed as a ratio or percentage. 